\title{
Prevención, promoción del desarrollo y atención temprana en la Escuela Infantil
}

\section{Prevenção, promoção do desenvolvimento e atenção precoce na Escola Infantil}

\section{Prevention, promotion of development and early intervention in the Nursery School}

\author{
Julio Pérez-López ${ }^{1}$ \\ María Teresa Martínez-Fuentes ${ }^{2}$ \\ Ángela Díaz-Herrero ${ }^{3}$ \\ Alfredo G. Brito de la Nuez ${ }^{4}$
}

\begin{abstract}
RESUMEN
En este trabajo se plantea la importancia y necesidad, desde el ámbito de la atención temprana, de poner en marcha programas de prevención primaria orientados a promocionar el desarrollo infantil y el ajuste familiar y escolar. Se expone la experiencia llevada a cabo desde el año 2005 por el Grupo de Investigación en Atención Temprana (GIAT) de la Universidad de Murcia (España), y se analizan los resultados obtenidos en el curso 2010-2011 en la Escuela Infantil de Lorquí (Murcia, España) con un grupo de 64 niños y sus respectivas familias. Los resultados indican un progreso mental y psicomotor de los niños, entre el principio y el final del curso escolar, lo que pone en evidencia la relevancia de este tipo de programa. También se constata un mejor nivel de desarrollo inicial en los niños y las familias
\end{abstract}

\footnotetext{
${ }^{1}$ Doctor en Psicología. Profesor de la Universidad de Murcia, España. E-mail: juliopl@um.es.

${ }^{2}$ Doctora en Psicología. Profesora de la Universidad de Murcia, España. E-mail: mtmartin@ um.es.

${ }^{3}$ Doctora en Psicología. Profesora de la Universidad de Murcia, España. E-mail: adiaz@um.es.

${ }^{4}$ Doctor en Psicología. Profesor de la Universidad de Murcia, España. E-mail: abrito@um.es.

Profesores del Grupo de Investigación en Atención Temprana (GIAT) - Universidad de Murcia (España).
} 
que han seguido el programa de prevención y promoción del desarrollo infantil frente a los que no lo han recibido.

Palabras-clave: atención temprana; prevención primaria; promoción del desarrollo infantil; educación infantil; Escuela Infantil.

\title{
RESUMO
}

Este artigo evidencia a importância e a necessidade de implementar, no âmbito da atenção precoce, programas de prevenção primária destinados a promover o desenvolvimento infantil e o ajuste familiar e escolar. Descreve a experiência realizada desde 2005 pelo Grupo de Pesquisa em Atenção Precoce (GIAT), da Universidade de Murcia (Espanha), e analisa os resultados obtidos nos anos de 2010-2011 na Escola Infantil de Lorquí (Murcia, Espanha), com um grupo de 64 crianças e suas respectivas famílias. Os resultados indicam um progresso no desenvolvimento mental e psicomotor das crianças, entre o início e o final do curso escolar, o que evidencia a relevância deste tipo de programa. Também constata um melhor nível de desenvolvimento inicial nas crianças e famílias que frequentaram o programa de prevenção e promoção do desenvolvimento infantil frente aos que não o frequentaram.

Palavras-chave: atenção precoce; prevenção primária; promoção do desenvolvimento infantil; educação infantil; creche.

\begin{abstract}
This paper discusses the importance and necessity from the field of early childhood, to implement primary prevention programs designed to promote child development and family and school adjustment. It describes the experiment carried out since 2005 by the Research Group on Early Childhood Intervention (GIAT), University of Murcia (Spain), and analyzed the results of the 2010-2011 academic years in the Infant School Lorquí (Murcia, Spain) with a group of 64 children and their families. The results indicate an improvement of mental and psychomotor development of children, between the beginning and the end of the school, which highlights the relevance of such services. The study finds a higher level of initial development in children and families who have followed the program of prevention and promotion of child development compared to those who have not received it.
\end{abstract}

Keywords: early intervention; primary prevention; promoting child development; early childhood education; Nursery School. 


\section{Introducción}

A lo largo de los últimos años, la atención temprana se ha ido transformando en un concepto integrador de las actuaciones sociales dirigidas a garantizar la igualdad de oportunidades de los niños con discapacidades o en situaciones de riesgo biopsicosocial y sus familias. En el contexto del cambio social y de los enfoques que dan primacía al desarrollo personal y la participación social, la aparición de nuevos escenarios de intervención (prenatal, neonatal, domicilio, guarderías, escuelas) ha convertido la atención temprana en una actividad muy compleja en la que resulta imprescindible el trabajo en equipo, la colaboración con la familia y con otros recursos sociales. Al tiempo, ha puesto de actualidad la necesidad de revisar y sistematizar las prestaciones extraordinarias que las distintas administraciones públicas tienen que aportar a los niños y sus familias para que tomen parte activa en la vida social.

El Servicio de Prevención, Promoción del Desarrollo Infantil y Atención Temprana (SEPRODIAT) de la Universidad de Murcia se creó como tal en noviembre de 2004. Surgió ante la demanda de 60 familias que participaron en un proyecto de investigación iniciado en enero del año 2001 y finalizado en diciembre del 2003. Dicho proyecto contemplaba el seguimiento de 60 niños desde el mes después del nacimiento hasta los 3 años de vida. Estos padres destacaron la utilidad, sobre todo de tipo preventivo, de este tipo de servicio. Para estos padres, especialmente los primerizos, supuso una fuente de información y asesoramiento extremadamente valiosa, ya que les transmitía seguridad en su papel de padres y también servía para detectar muy precozmente cualquier alteración en el desarrollo de sus hijos.

Con estas referencias, se planteó a las autoridades académicas de la Universidad de Murcia (UMU) la conveniencia de poner en marcha un servicio de estas características y en noviembre de año 2004 se iniciaron las primeras evaluaciones y orientaciones desde el Servicio de Instrumentación Psicológica ubicado físicamente en el entonces denominado Servicio de Apoyo a las Ciencias Experimentales, y hoy llamado Servicio de Apoyo a la Investigación de la Universidad de Murcia.

Los supuestos teóricos y sociales en los que se basa este Servicio son los expuestos en el Libro Blanco de la Atención Temprana (GAT, 2000), fundamentalmente en los referidos a la prevención primaria y secundaria en Atención Temprana. 


\section{Finalidad del SEPRODIAT}

Se pretende ofrecer un servicio que sirva para prevenir y promocionar el desarrollo de los niños desde sus primeros años de vida y para favorecer el ajuste familiar y escolar. En este sentido cabe mencionar que la familia es considerada como uno de los contextos de desarrollo más importantes para los niños que componen esa estructura, y juega un papel muy relevante en el proceso de potenciación del desarrollo de sus miembros. Pero también consideramos que el contexto de la Escuela Infantil es esencial para completar el desarrollo de los niños y el ajuste de las familias.

\section{Justificación del SEPRODIAT}

Como ya destacábamos en un trabajo anterior (PÉREZ-LÓPEZ; MARTÍNEZ-FUENTES; DÍAZ-HERRERO; BRITO DE LA NUEZ, 2006), creemos y defendemos que la atención a la infancia, por representar un momento trascendental en la vida de la persona, debe ser considerada de forma prioritaria. En este sentido, desde las Entidades Públicas se llevan a cabo, desde hace tiempo, programas y acciones específicas encaminadas a promocionar y proteger la salud del niño. El programa de atención al niño sano en la Región de Murcia es un claro ejemplo de estas actuaciones. No obstante, echamos en falta la prevención y la promoción en un área tan importante como es el desarrollo psicológico y social en los primeros años de vida del niño.

Para intentar cubrir, en la medida de lo posible, estas necesidades es por lo que surgió el Servicio de Prevención, Promoción del Desarrollo Infantil y Atención Temprana (SEPRODIAT). Con este servicio se pretende:

Dar respuesta a necesidades detectadas, pero tratando de no competir con los servicios ya existentes y complementar los ámbitos que dichos servicios no alcanzan; siendo sus prioridades: la Prevención y Promoción del Desarrollo Infantil; la atención preferente para los hijos de miembros de la "comunidad universitaria"; y la especial atención al desarrollo de trabajos de investigación en este ámbito.

Tener un "Marco teórico" de referencia, contrastado e innovador. Se enmarca en las corrientes más innovadoras y de resultados ya contrastados: las teorías y la 
investigación sobre el desarrollo; los nuevos enfoques de la atención temprana; el tratamiento de las necesidades educativas especiales, en coordinación con la Asociación de Profesionales de Atención Temprana de la Región de Murcia (ATEMP).

Estar sometido a un "Marco legislativo" que favorece y promueve este tipo de Servicios: Normativa de ámbito estatal: Ley de Dependencia, LOGSE y LOE; Normativa regional (Decreto de mínimos para los Centros de Atención a la Infancia (CAI) y los Puntos de Atención a la Infancia (PAI); Proyecto de Decreto de mínimos para los Centros de Desarrollo Infantil y Atención Temprana (CDIAT); Normas de la Universidad de Murcia; Experiencias en otras Universidades del Estado e Internacionales (la Universidad de Valencia, el Centro Nacional de Defectos Congénitos y Deficiencias del Desarrollo de Atlanta, y el National Institute of Neurological Disorders and Stroke de Bethesda, en Estados Unidos, pueden servir de ejemplo).

Tener un proyecto con un Enfoque globalizador $(I+D+I)$ e interdepartamental: planteamiento coordinado y complementario al Centro de Atención a la Infancia o Escuela Infantil de la Universidad de Murcia; coordinación estrecha y preferente con los servicios públicos existentes en atención temprana y Educación Infantil, como los Equipos de Atención Temprana de la Comunidad Autónoma de Murcia; los CDIAT Municipales (Ayuntamientos y Consejería de Sanidad y Política Social). La finalidad de esta coordinación es complementar tareas de apoyo y tratamiento; elaborar programas de prevención y desarrollar trabajos de investigación de ámbito regional.

\section{Objetivos y actividades del SEPRODIAT}

El SEPRODIAT (Servicio de Prevención, Promoción del Desarrollo Infantil y Atención Temprana) intenta potenciar las capacidades de desarrollo y bienestar de los niños desde el nacimiento hasta los tres años; orientar y asesorar a los padres y madres en las tareas de crianza; favorecer la adaptación mutua de la familia y el niño, así como su autonomía; detectar, lo más precozmente, cualquier riesgo o alteración del desarrollo; y derivar, a los niños y sus familias, a los servicios oportunos que existen en nuestra comunidad autónoma.

De forma más concreta se realizan las siguientes funciones: 
Prevención: sensibilización de la población en general en aspectos de prevención relacionados con el desarrollo infantil, la formación a las familias y futuros padres/madres, y la coordinación y colaboración de los profesionales de las áreas sanitaria, educativa y social.

Intervención: (a) valoración inicial de los casos, con estudio global y profundo del desarrollo del niño, de su historia individual y familiar y de su entorno. Esta función incluye la acogida familiar, la recogida de información; la evaluación del niño y de su entorno; la elaboración de hipótesis diagnósticas; la elaboración del plan de intervención; y la entrevista de devolución diagnóstica a la familia; (b) atención a la familia, informándoles sobre los diferentes recursos disponibles, apoyándoles en la adaptación a las características de su hijo, o derivándoles a los servicios pertinentes en los casos en que así se precise; (c) intervención en el entorno de la Escuela Infantil, diseñando estrategias de adaptación al medio físico y social en función de las necesidades de los niños y proporcionando información a los educadores del Centro sobre las estrategias educativas más adecuadas en función de las características valoradas en los niños.

Coordinación y derivación: (a) coordinación con el sistema educativo para la atención adecuada a los usuarios de Atención Temprana integrados en aquel sistema; (b) coordinación con el sistema sanitario para atención a los niños; (c) derivación a otros servicios asistenciales, si procede.

Otros objetivos específicos que se llevan a cabo son: (a) desarrollar y fomentar actividades de Investigación, Desarrollo e Innovación (I+D+I) en las áreas de conocimiento relacionadas con el tratamiento y la atención temprana en todos sus ámbitos; (b) facilitar a los jóvenes graduados la iniciación y formación en el trabajo y la investigación en dichas áreas, a través de la realización de prácticas en enseñanzas de segundo y tercer ciclo integradas y organizadas para los postgrados y programas de doctorado; (c) fomentar y promover las relaciones de intercambio o cooperación, en materias de formación e investigación que le son propias en su conexión con las áreas afines, con otros centros ubicados en la Comunidad Autónoma de la Región de Murcia, en España o en el extranjero; (d) fomentar y difundir la importancia de la prevención y promoción del Desarrollo Infantil a la sociedad, las empresas y organismos de los sectores relacionados con la discapacidad.

Con estos objetivos, las actividades realizadas en el servicio que se llevan a cabo son: (a) evaluación y seguimiento del desarrollo del niño a las edades de uno, seis, doce, dieciocho, veinticuatro y treinta y seis meses; (b) evaluación del tipo de vinculación afectiva a los quince meses; (c) asesoramiento a las familias sobre pautas de educación y crianza, basadas en las características del desar- 
rollo de sus hijos; (d) detección precoz de posibles alteraciones del desarrollo o de la interacción adulto-niño; (e) derivación, en caso de ser necesario, a los servicios pertinentes.

\section{Beneficiarios y actividades desarrolladas}

La prevención y la atención temprana en este servicio están dirigidas a todos los niños en general y especialmente a aquellos que presentan cualquier tipo riesgo o de trastorno en el desarrollo y sus familias.

Se presta servicio a los niños de 0 a 3 años que pertenecen, prioritariamente, a familias vinculadas a la comunidad universitaria, pero también de fuera de ella; y a los niños que asisten a las Escuelas Infantiles que hayan establecido un contrato o convenio con la Universidad de Murcia.

Desde su inicio hasta mayo de 2011 se han evaluado en el Servicio a 374 niños, cuyo seguimiento ha supuesto más de 1488 evaluaciones.

La coordinación que se lleva a cabo con los Equipos de Atención Temprana de Murcia, que dependen de la Consejería de Educación de la Comunidad Autónoma de la Región de Murcia., nos ha permitido derivar 18 niños en los que se detectaron necesidades de intervención, y se estableció contacto con los Centros de Desarrollo Infantil y Atención Temprana Municipales y con Centros pertenecientes a Asociaciones, para transmitirles información sobre la derivación de los niños y sus familias cuando se detectó algún factor de riesgo importante o patología concreta con el fin de que pusieran en marcha el proceso de intervención más adecuado.

\section{Prevención y promoción del desarrollo en las Escuelas Infantiles}

Con la Escuela Infantil de Lorquí, se lleva a cabo un "Programa de prevención y promoción del desarrollo infantil" desde el curso 2005-2006 que continúa en la actualidad, a través de un contrato entre el Ayuntamiento de Lorquí y la Universidad de Murcia, en el que se han realizado las siguientes actividades:

- Evaluación y seguimiento del desarrollo de todos aquellos niños escolarizados y cuyos padres firmaron el consentimiento informado para participar. Se efectúan dos evaluaciones, la primera, se realiza en el mes de octubre-noviembre y la segunda en el mes de mayo-junio. 
- Asesoramiento a las familias sobre características del desarrollo de sus hijos, proporcionándoles pautas específicas y adaptadas a las características de sus hijos con el fin de fomentar su desarrollo y prevenir posibles problemas en los niños. - Tres charlas formativas a padres y madres a lo largo del curso. Una sobre importancia interacciones adulto niño para promocionar el desarrollo infantil, otra sobre la importancia del juego para el desarrollo y una tercera sobre cómo abordar las conductas disruptivas y las rabietas en los niños.

- Seminarios o talleres de formación a los profesionales de la Escuela Infantil en función de sus demandas o de las necesidades detectadas en los niños y las familias.

- Detección precoz de posibles alteraciones del desarrollo o de la interacción adulto-niño,

- Derivación, en caso de ser necesario, a los servicios pertinentes.

Desde el curso 2008-2009 hasta la actualidad, fue la propia Universidad de Murcia quien solicitó los Servicios del "Programa de prevención y promoción del desarrollo infantil" para llevarlo a cabo en el Centro de Atención a la Infancia ubicado en el campus universitario de Espinardo.

\section{Beneficiarios y actividades desarrolladas en la Escuela Infantil}

Desde octubre de 2005 hasta junio de 2011 se ha realizado, en la Escuela Infantil de Lorquí, el seguimiento de 382 niños, con un total de 764 evaluaciones y 382 entrevistas con los padres.

En la Escuela Infantil de la Universidad de Murcia se ha realizado, desde noviembre de 2008 hasta junio de 2011, el seguimiento de 175 niños, con un total de 350 evaluaciones.

En total, desde la puesta en marcha del "Programa de prevención y promoción del desarrollo infantil" se han seguido a 557 niños y se han efectuado 1114 evaluaciones. De todos los niños evaluados, 34 fueron derivados a los Centros de la Comunidad Autónoma para que se les elaborara un programa de intervención dado que presentaban diferentes patologías del desarrollo, a $75 \mathrm{se}$ les dieron pautas específicas para fomentar el desarrollo bien en el aula o bien en casa. Al resto se les proporcionaron orientaciones, en función de las características de los niños, para seguir promocionando su desarrollo, pero siempre dentro de actividades de juego y aprovechando las rutinas de la vida cotidiana. No se trata de convertir la casa en una escuela.

Otras actividades realizadas son: - colaboración en el desarrollo de un programa de prevención del Desarrollo Infantil con algunos servicios de preparación al parto de algunos centros de salud de Alicante; - hemos actuado como Centro de segunda opinión para aquellos padres cuyos hijos estaban siendo 
atendidos por otros CDIAT's; - colaboración con la Asociación de Profesionales de Atención Temprana de la Región de Murcia (ATEMP) mediante convenio firmado entre las partes; - participamos en el Practicum de la Licenciatura de Psicología de Universidad de Murcia y Universidad Miguel Hernández de Elche; - participamos en el Practicum del Master Oficial de Psicología de la Educación de la Facultad de Psicología de la Universidad de Murcia desde el curso 2009-2010; - participamos en las Prácticas correspondientes al Master en Atención Temprana que imparte la Universidad de Murcia como título de estudios propios; - efectuamos acciones de formación con alumnos internos del Departamento de Psicología Evolutiva y de la Educación.

\section{A modo de conclusión}

Resumir en unas pocas líneas toda la labor desarrollada por el SEPRODIAT a lo largo de estos años ha resultado complicado, pero expresar la ilusión y convicción de estos profesionales en el desarrollo de la Atención Temprana aún resulta más difícil. Pensamos que con este Servicio se contribuye a la implantación y la mejora de la Atención Temprana en la Región de Murcia en una parcela que para la mayoría de los Centros de Desarrollo Infantil y Atención Temprana (CDIAT) resulta difícil llegar como es la prevención primaria.

Por último, también queremos destacar dos rasgos distintivos que avalan al grupo de profesores y profesionales que forman el SEPRODIAT. En primer lugar, que todas estas actividades se han realizado de una manera totalmente altruista y desinteresada por parte de los componentes del Servicio. En segundo lugar, las actividades realizadas han sido un trabajo añadido a las tareas de docencia, investigación y gestión propias de las universidades españolas.

\section{Método}

Para comprobar si este tipo de servicios resulta efectivo, presentaremos a continuación los resultados obtenidos con los niños evaluados en la Escuela Infantil de Lorquí (Murcia) a lo largo del curso 2010-2011. Nuestro propósito radica en responder a dos objetivos. El primero, si este tipo de programas de intervención es eficaz y, en segundo lugar, si existen diferencias en cuanto al 
nivel de desarrollo en niños que ya han seguido el programa frente a niños que se escolarizan por primera vez.

\section{Participantes}

En el curso académico 2010-2011, se ha realizado la valoración y seguimiento del desarrollo de 34 niñas y 30 niños cuyas familias firmaron el consentimiento informado.

En la primera evaluación la media de edad fue de 25 meses 3 días (edad mínima 12 meses 1 día y máxima de 35 meses 5 días). En la segunda evaluación, la media de edad fue de 31 meses 9 días (edad mínima 17 meses 21 días y edad máxima 41 meses 18 días).

En la primera evaluación se realizaron 73 valoraciones del desarrollo y en la segunda fueron 71. En total hemos revisado a 75 niños diferentes con sus respectivas familias, pero solo 64 fueron valorados en las dos ocasiones. Los niños a los que sólo se les efectuó una valoración fue debido a que tuvieron altas en la Escuela Infantil después de los momentos mencionados, o fueron dados de baja en el Centro. En total se han efectuado 144 valoraciones del desarrollo.

De los 64 niños evaluados, 20 ya habían asistido a la Escuela Infantil el año anterior, y por tanto habían participado en el "programa de prevención, promoción del desarrollo infantil", y 44 niños eran escolarizados por primera vez.

\section{Instrumentos}

Para efectuar la evaluación del desarrollo se utilizaron las Escalas Bayley del Desarrollo Infantil (BSID-II), en su segunda edición (BAYLEY, 1993). Estas escalas nos proporcionan un Índice de Desarrollo Mental - IDM - (que nos indica la agudeza sensorio-perceptiva, la discriminación y capacidad de respuesta a estímulos, la adquisición temprana de la permanencia del objeto y de la memoria, el aprendizaje y la capacidad de resolución de problemas, las vocalizaciones al comienzo de la comunicación verbal, y la capacidad temprana para generalizar y clasificar); y un Índice de Desarrollo Psicomotor - IDP (donde se mide el grado de control del cuerpo, la coordinación de los músculos grandes y la habilidad manipulativa de manos y dedos de los niños). En ambos casos, el valor promedio es 100, y la desviación típica de 15 . 


\section{Procedimientos}

Todos los niños y niñas participantes en este estudio fueron evaluados en dos ocasiones. La primera evaluación se llevó a cabo, desde el mes de noviembre de 2010 hasta enero de 2011, y la segunda evaluación durante los meses de mayo y junio de 2011 en la Escuela Infantil de "Lorquí" (Murcia, España). En ambas ocasiones estuvieron presentes los padres y/o madres de los niños, a los que se les daban pautas para el buen desarrollo de sus hijos. Los profesionales del Centro eran los que se encargaban de establecer los días y hora de cada evaluación. A lo largo del citado curso se impartieron dos charlas para los padres y madres de los niños que participaban en el programa de prevención. La primera sobre la importancia de las interacciones adulto-niño para fomentar el apego y el desarrollo infantil, y la segunda sobre la importancia del juego y el tipo de juegos y juguetes más adecuados para cada edad. La primera charla se impartió en el mes de noviembre y la segunda en el mes de diciembre de 2010.

\section{Resultados}

Todos los datos fueron analizados con el paquete estadístico informatizado SPSS 15.0.

En cuanto a los valores globales del desarrollo tanto en la primera como en la segunda evaluación, desde un punto de vista descriptivo, debemos destacar que, a nivel general, los niños que han sido evaluados en la Escuela Infantil de Lorquí durante el curso 2010-2011, se sitúan dentro de los niveles esperados de desarrollo (véase Gráfica 1).

$\begin{array}{ll}\text { IDM promedio } & \text { primera evaluación 103,32 } \\ & \text { segunda evaluación 109,87 } \\ \text { IDP promedio } & \text { primera evaluación 106,75 } \\ & \text { segunda evaluación 109,98 }\end{array}$



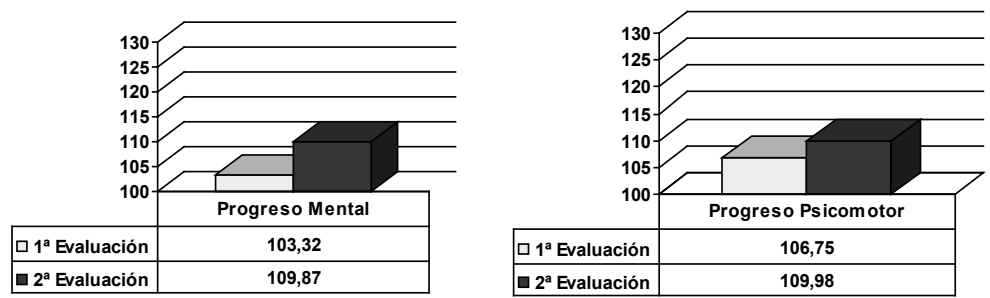

GRÁFICA 1 - Índices del desarrollo mental y psicomotor general de la Escuela Infantil de Lorquí. Curso 2010-2011. Con todos los niños. FONTE: Los autores (2011)

Si hacemos un análisis detallado del progreso mental y psicomotor general en cada momento de evaluación también podemos observar una mejora evidente entre la primera y la segunda valoración del desarrollo.

Debemos aclarar que el progreso mental y psicomotor se refiere a si existe alguna mejoría en estos ámbitos, sin que ésta dependa directamente de un índice de desarrollo. En este sentido, se comparan los resultados del niño en relación consigo mismo a lo largo de los distintos momentos temporales. Ello nos permitirá disponer de una medida que nos dé información acerca de si existe o no una evolución adecuada a nivel individual.

Para responder a esta idea hemos utilizado una variable a la que denominamos progreso (BRITO DE LA NUEZ; DÍAZ-HERRERO; PÉREZ-LÓPEZ; MARTÍNEZ-FUENTES; SÁNCHEZ-CARAVACA, 2004; PÉREZ-LÓPEZ; SÁNCHEZ-CARAVACA, 2008). Ésta se obtiene de la diferencia, en cada niño, entre la Edad Equivalente de Desarrollo (EED) en la escala mental o en la escala psicomotora y la Edad Cronológica (EC) dividido por la edad (EC) y multiplicado por cien, tal como se indica en la siguiente fórmula:

$$
\text { Progreso }=\frac{\text { EED }- \text { EC }}{\text { EC }} \times 100
$$

Las puntuaciones obtenidas mediante este procedimiento deben interpretarse de la siguiente forma: (a) Un valor positivo indica que la edad de desarrollo del niño es superior a la edad en la que se efectuó la medida. (b) Un valor negativo indica que la edad de desarrollo es inferior a la edad en la que se efectuó la medida. (c) Un valor próximo a cero sugiere que la edad de desarrollo del niño se aproxima a la edad en que se efectuó la medida.

A nivel general los datos obtenidos han sido muy favorables como puede apreciarse en las gráficas siguientes. En concreto, en el área mental se ha pro- 
ducido una mejora desde la primera hasta la segunda valoración, situándose el valor promedio del desarrollo mental 8,21 puntos por encima del valor esperado para su edad cronológica. En el área psicomotora también se han producido mejoras, situándose el valor promedio en 8,33 puntos por encima de la media esperada para la edad.
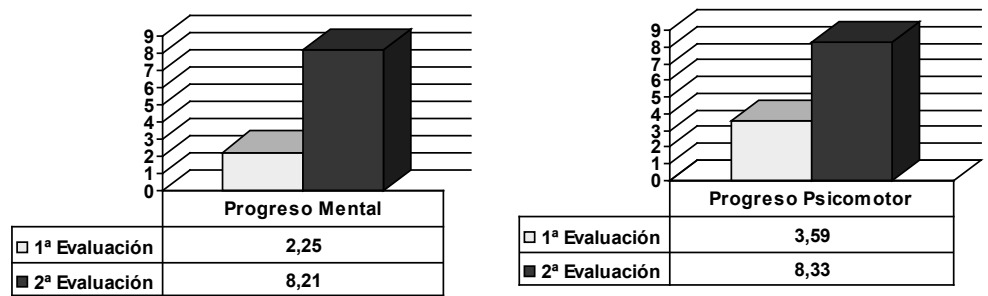

GRÁFICA 2 - Progreso mental y progreso psicomotor general de la Escuela Infantil de Lorquí. Curso 2010-2011. Con todos los niños. FONTE: Los autores (2011)

Efectuamos pruebas $\mathrm{T}$ de Student para muestras relacionadas, con el fin de comprobar si estas diferencias eran significativas y los resultados confirman la significación para el área mental $\left(\mathrm{T}_{63}=-5,95 ; \mathrm{p}=.000\right)$, y para el área psicomotora $\left(\mathrm{T}_{59}=-4,73 ; \mathrm{p}=.004\right)$.

Para comprobar el segundo objetivo, en el curso 2010-2011 valoramos, en diferentes aulas, a 20 niños que ya asistieron a la Escuela Infantil durante el curso anterior, y por tanto, que ya habían seguido el programa de prevención y promoción del desarrollo infantil, frente a 44 niños que se habían matriculado por primera vez en el centro. A continuación presentamos una comparación en cuanto al nivel de progreso, tanto mental como psicomotor, de ambos grupos. Como puede observarse en las gráficas, los niños que asistieron a la escuela infantil durante el curso anterior han obtenido, en la primera evaluación, puntuaciones más altas en el progreso mental y en el psicomotor que los niños que asisten por primera vez al centro. Para comprobar si estas diferencias eran significativas desde un punto de vista estadístico, realizamos pruebas T de Student para muestras independientes. Los resultados obtenidos fueron $\mathrm{T}_{63}=-2,19(\mathrm{p}=.030)$ para el progreso mental y $\mathrm{T}_{59}=-0,943(\mathrm{p}=.349)$ para el progreso psicomotor, es decir, solo existían diferencias significativas en cuanto al progreso mental.

Con los resultados obtenidos en la segunda evaluación, repetimos el tipo de análisis, pero no se alcanzó significación estadística en el progreso de ninguna de las dos escalas. 

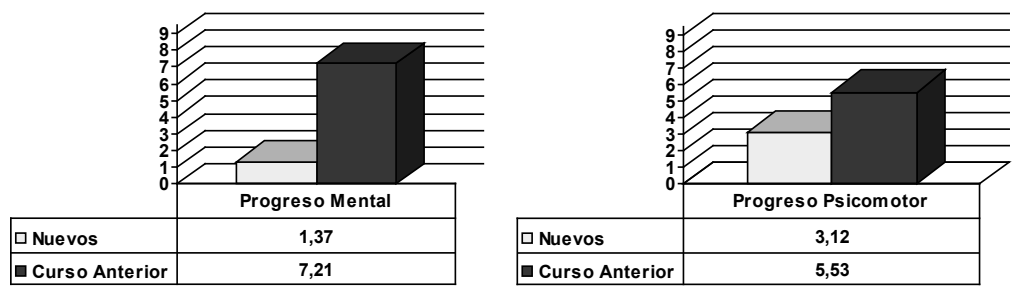

GRÁFICA 3 - Comparación del progreso de niños nuevos y de niños que asistían al centro el curso anterior en la primera evaluación.

FONTE: Los autores (2011)
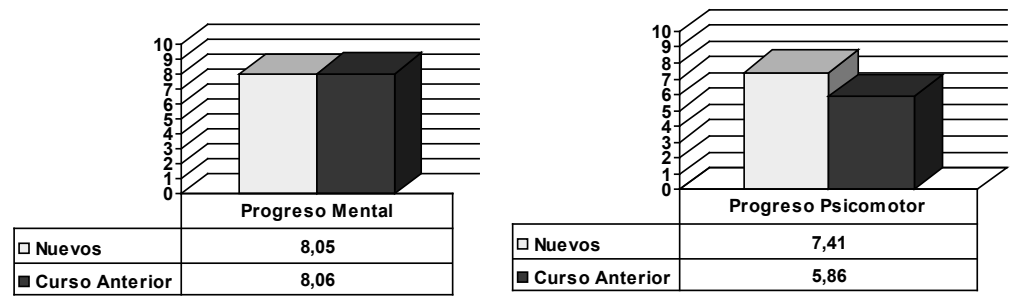

GRÁFICA 4 - Comparación del progreso de niños nuevos y de niños que asistían al centro el curso anterior en la segunda evaluación.

FONTE: Los autores (2011)

\section{Discusión y conclusiones}

Los resultados globales correspondientes al curso 2010-2011 nos muestran un progreso significativo entre la primera y la segunda evaluación, tanto en el área mental como en la psicomotora en los niños que fueron seguidos a través del "Programa de prevención y promoción el desarrollo". Estos resultados, nos permiten afirmar que este tipo de intervención supone una modalidad de prevención eficaz especialmente en el ámbito escolar y familiar, al suministrar, tanto a progenitores como a educadores, información, apoyo y asesoramiento que les prepara para proporcionar a los niños las mejores condiciones posibles de crianza en un contexto específico. Conclusiones que coinciden con los trabajos de CANDEL (2004); DÍAZ-HERRERO; MARTÍNEZ-FUENTES, (2009).

Además, nuestros resultados también indican que aquellos niños y familias que ya participaron en el programa de prevención a lo largo curso anterior, 
presentan mejores condiciones iniciales, en este segundo año, a nivel mental que los niños de nueva escolarización. La no existencia de diferencias en el área psicomotora puede ser debida a que ésta está más influenciada por factores de maduración y práctica.

Por otra parte, el hecho de que en la segunda evaluación no existan diferencias significativas entre el grupo de niños nuevos y el grupo de niños del curso anterior, no hace más que constatar de nuevo la eficacia del programa de prevención y promoción del desarrollo infantil.

En resumen, nuestros resultados ponen de manifiesto la eficacia de este tipo de servicios que sirven para prevenir y promocionar el desarrollo de los niños desde sus primeros años de vida y para favorecer el ajuste familiar y escolar. Es decir, la eficacia de la prevención primaria en las Escuelas Infantiles desde el enfoque de la Atención Temprana.

\section{REFERENCIAS}

BAYLEY, N. Bayley Scales of Infant Development. Second Edition (BSID-II). San Antonio. Hartcourt Brace \& Company, 1993.

BRITO DE LA NUEZ, A. G.; DÍAZ-HERRERO, A.; PÉREZ-LÓPEZ, J.; MARTÍNEZ-FUENTES, M. T.; SÁNCHEZ-CARAVACA, J. Tendencias del desarrollo mental en el primer año de vida: un análisis comparativo en niños nacidos a término y prematuros. Comunicación presentada al II Congreso Internacional de Atención Temprana: Atención temprana en el Siglo XXI: Nuevos retos, Santiago de Compostela, 2004.

CANDEL, I. Prevención desde el ámbito educativo: patologías no evidentes. In: PÉREZLÓPEZ, J.; BRITO DE LA NUEZ, A. G. (Ed.). Manual de atención temprana. Madrid: Pirámide, 2004, p. 119-132.

DÍAZ-HERRERO, A.; MARTÍNEZ-FUENTES, M. T. Prevención y promoción del desarrollo infantil: una experiencia en las escuelas infantiles. Revista Interuniversitaria del Profesorado, n. 65 (23,2), p. 57-72, 2009.

GAT. Libro Blanco de la Atención Temprana. Madrid: Real Patronato de Prevención y de Atención a Personas con Minusvalía, 2000.

PÉREZ-LÓPEZ, J.; BRITO DE LA NUEZ, A. G. (Ed.). Manual de atención temprana. Madrid: Pirámide, 2004.

; MARTÍNEZ-FUENTES, M. T.; DÍAZ-HERRERO, A.; BRITO DE LA NUEZ, A. Prevención, promoción del desarrollo infantil y atención temprana. Revista de Atención Temprana, n. 9, p. 77-84, 2006. 
; SÁNCHEZ-CARAVACA, J. Riesgo y tratamiento como factores responsables del progreso en los niños prematuros. In: GONZÁLEZ-PIENDA, J. A.; NÚÑEZ-PÉREZ, J. C. (Ed.). Psicología y Educación: un lugar de encuentro. Oviedo: Ediciones de la Universidad de Oviedo, 2008. p. 1631-1640.

Texto recebido em 29 de agosto de 2011 .

Texto aprovado em 31 de outubro de 2011. 